Title:

Diffusive Transport in Modern Polymeric Materials

Submitted to:

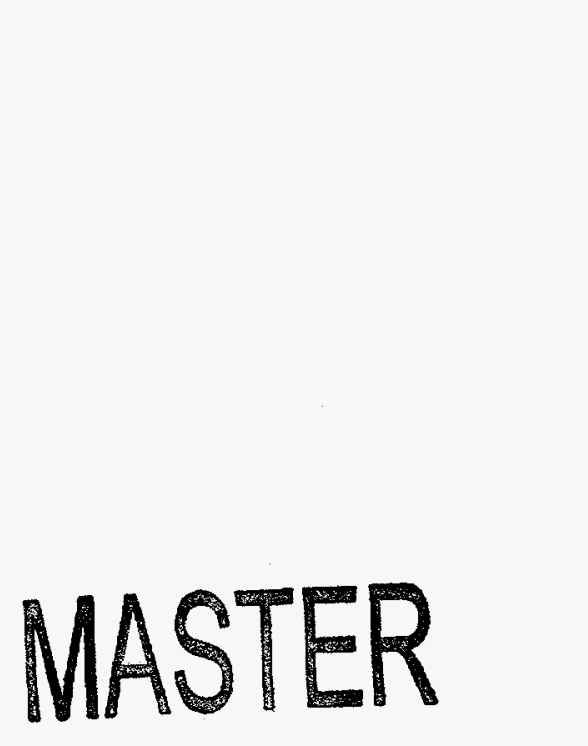

DOE Office of Scientific and Technical Information (OSTI)

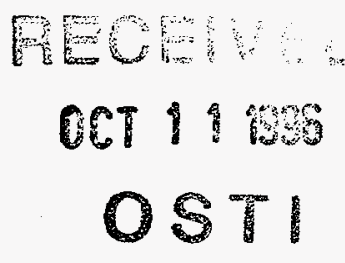

Author(s):

Charles Doering, CNLS

$0 . \mathrm{TI}$

Los Alamos

NATIONAL LABORATORY

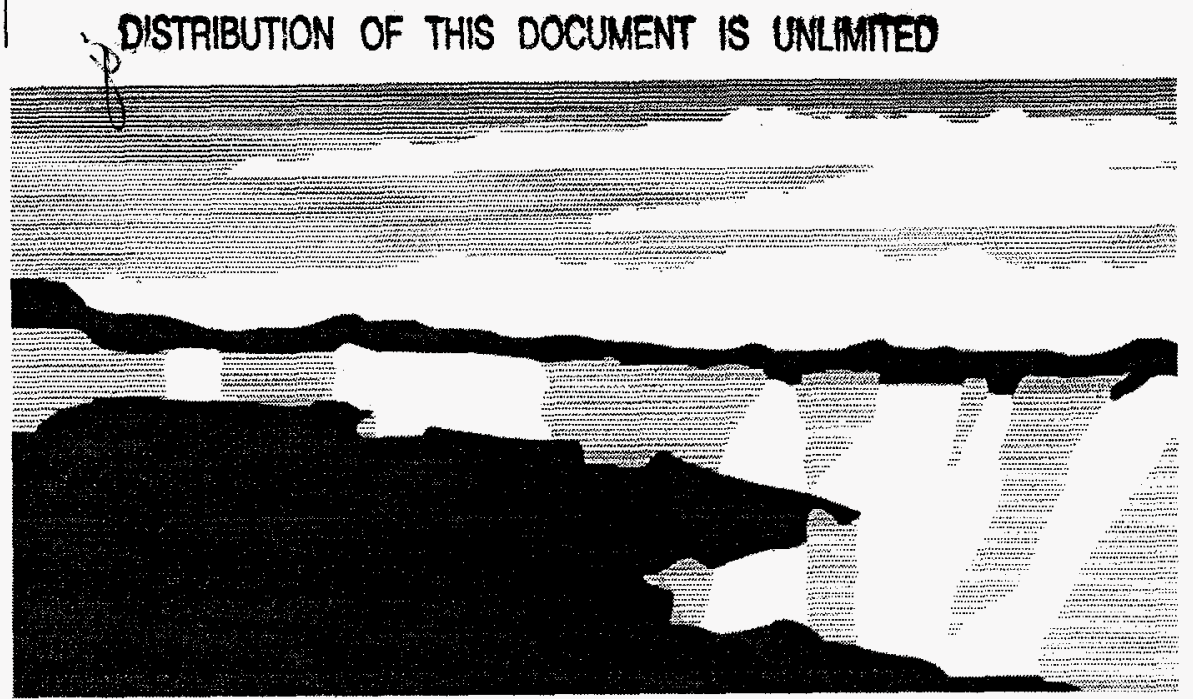

Los Alamos National Laboratory, an affirmative action/equal opportunity employer, is operated by the University of California for the U.S. Department of Energy under contract W-7405-ENG-36. By acceptance of this article, the publisher recognizes that the U.S. Government retains a nonexclusive, royaltyfree license to publish or reproduce the published form of this contribution, or to allow others to do so, for U.S. Government purposes. The Los Alamos National Laboratory requests that the publisher identify this article as work performed under the auspices of the U.S. Department of Energy. 


\section{DISCLAIMER}

Portions of this document may be illegible in electronic image products. Images are produced from the best available original document. 


\title{
Diffusive Transport in Modern Polymeric Materials
}

Charles Doering*, Martin Bier (University of Chicago), Kostas Christodoulou

(DuPont), Chris Durning (Columbia University), David Edwards (New York

University), Larry Eisenberg (Risk Control Corporation), Thomas Erneux

(Universite Libro de Bruxelles), Ben Lee (University of Maryland), Michael

Louie (California Institute of Technology), Aleksei Makhankov (Joint Institute

for Nuclear Research), Peter Palffy-Muhoray (Kent State University), Luis

Reyna (IBM Watson Research Center), Joseph Watkins (University of

Arizona), and Shanji Xiong (Columbia University)

\begin{abstract}
This is the final report of a one-year, Laboratory-Directed Research and Develpoment (LDRD) project at the Los Alamos National Laboratory (LANL). Polymers, composites, and synthetic modern materials are replacing traditional materials in many older scientific, engineering, commercial, and military applications. This project sought to focus on the new polymeric materials, deriving and analyzing models that predict their seemingly mysterious transport properties. It sought to identify the dominant physical mechanisms and the pertinent dimensionless parameters, produce viable theoretical models, and devise asymptotic and numerical methods for use in specific problems.
\end{abstract}

\section{Background and Scientific Approach}

Wholly new devices, applications, and even entire new fields are emerging because of the existence of these new materials. New adhesives, bonding materials, and sheet molding compounds offer great gains in strength and flexibility and savings in weight when used as critical materials in commercial and military vehicles and devices. "Smart" polymer gels will provide the basis for innovative and sophisticated devices for use in drug delivery, agriculture, environmental chemistry, and environmental protection and clean-up. Polymer films are the basis for

\footnotetext{
* Principal investigator, e-mail: doering@lanl.gov
} 
protective clothing, uniforms, and membranes designed for use with harmful gases and toxic chemicals. Pharmaceuticals will be revolutionized by appropriately combining drugs with "designer" polymers for controlled release and specifically targeted use. The aging and failure of composites (both accidentally and more importantly in a controlled manner) will become a precise application. For the past few years, national and international chemical, chemical engineering, and materials sciences societies have devoted many sessions to a truly dazzling array of common and exotic uses and problems [1-6]. It is important to have valid (hopefully easily applicable) theories for the behavior of these materials and accurate control of their properties. Central problems emerging in these new technologies are types of diffusive motion quite unlike anything predicted by classical diffusion theory.

It is important to realize that for most of the new problems utilizing glassy polymers, although the problems are often described as diffusive, classical diffusion theory and its mathematical description cannot possibly describe the observations even when the diffusivity is a nonlinear function of the concentration. This is, of course, not surprising when one realizes that in addition to molecular diffusion, time-dependent diffusion processes in nonequilibrium glassy polymers involve many different fundamental phenomena at various levels, often including a phase transition from the glassy state to a rubbery state.

We are identifying the dominant physical mechanisms and the pertinent dimensionless parameters, producing viable theoretical models, and devising asymptotic and numerical methods for use in specific problems. The chief difficulty in deriving equations of motion is that the exact physical processes underlying the behavior of these materials are not well understood. Thus, any attempts to derive equations from first principles of physics (mechanics, thermodynamics, etc.) require postulates or assumptions at some level. However, the most important and most commonly mentioned properties of glassy polymers all stem from finite mechanical relaxation times resulting from slow response to changing conditions. These observations suggest that, although the interaction among the fundamental processes is not yet known, a global relaxation type phenomenon must be utilized in any physical basis for a model [7-16]. We have incorporated such mechanisms into our preliminary models, which essentially utilize physically reasonable partitions of the penetrant flux. The type of model is very satisfying in that it admits physically intuitive reasoning, it is easily applicable, and it is general enough to unify many diverse and complicated behavior patterns of the diffusing penetrant. The attempt is, then, partly physically sound and partly physically plausible, the plausibility being based on phenomenology and experimentally determinable parameters. Confidence is high because the models work in all cases so far $[5,6,17-22]$ and the mathematical structure used is strongly indicated by experiments and observations. We feel that if a true derivation from first principles (without assumptions) is ever possible, application 
to our problems will yield equations of the same form as we are using, and in fact, the few specific elementary applications that have been studied via fundamental principles are special cases of our theory. Our goal is the accurate description of penetrant concentration (for either sorption or desorption) and the basic physics involves serious consideration of the internal free energy and chemical potential and their dependence upon pertinent physical mechanisms.

The resulting equations, subject to various appropriate initial and boundary conditions at fixed and moving boundaries, constitute the mathematical basis for our problems involving diffusive transport in glassy polymers. These are highly nonlinear partial differential-integral equations and will require either nontrivial extension of existent numerical and asymptotic methods or the development of new ones.

\section{Importance to LANL's Science and Technology Base and National R\&D Needs}

This project supports a Los Alamos core competency in theory, modeling, and highperformance computing. The project enhances the Laboratory's visibility in modeling diffusive transport phenomena and enhances our ability to respond to initiatives requiring that competency.

\section{Scientific Approach and Results to Date}

Our approaches were initially developed in an attempt to account for so-called case II diffusion [11-15] in which sharp (shock-like) diffusive fronts form and propagate with constant speed, often yielding a steady-state concentration field with a jump discontinuity. The one-dimensional case (i.e., planar fronts) was studied in a series of papers [6-9] in which interesting asymptotic and numerical methods were developed and used successfully to account for the observations. An interesting development occurred in the doctoral research of $\mathrm{C}$. K. Hayes. In order to carry out a detailed perturbation analysis, certain difficulties continually arose which forced us to re-examine the derivation of traveling waves for the Fisher (or KPP) equation. In so doing, Hayes discovered a new class of traveling waves for that equation [23]; thus, enabling us to find similar waves for our initial model [17]. Some preliminary studies were also carried out for situations in which, in addition to the basic polymer-penetrant system, convection and chemistry at the penetration front takes place $[17,20]$. The chemical reaction mechanism accounts for the chemistry and partial interstitial immobilization of the penetrant molecules. 
The first two-dimensional problem was completed recently. D. S. Cohen, A. B. White, and T. P. Witelski [24] have developed a method for tracking the motion of the penetrant front. H. Amman of the University of Zurich became quite interested in our model and proved $[\mathbf{2 4 , 2 5}]$ the important result that shocks (discontinuities) occur only in the limit as time goes to infinity. Thus, extremely sharp gradients (but not discontinuities) form and propagate very early in time with the steady-state limit supporting a jump discontinuity. This very accurately reflects the physical reality in which the glass-rubber interface is not a strict thermodynamic phase change but instead occurs over some very small pertinent physical scale.

One of the most intriguing, dramatic, and difficult to explain anomalous phenomena is the appearance and propagation of multiple sharp (shock-like) diffusive fronts. These are of paramount importance in several applications where the entire device relies on the precise time history of the glass-rubber interface. Depending upon the polymer and penetrant, sometimes two or three sharp boundaries propagate with decreasing, constant or even increasing speed under various conditions. Detailed experimental observations have been made using optical methods in which one measures birefringence resulting from, for example, the swelling of cellulose acetate confined between parallel plates [1]. The penetrant is either water or chloroform in the liquid or vapor state. Two sharp fronts (for vapor penetrant) and three sharp fronts (for liquid penetrant) are observed. Sometimes the polymer is initially dry and sometimes the polymer initially contains a small amount of the penetrant uniformly distributed.

Since the experiments have not been done with any specific new model in mind, data are not available to find many of the properties of the functions and physical parameters in the equations or to verify conditions at moving boundaries. Furthermore, techniques do not even exist to measure some aspects of the phenomena directly, particularly at glass-rubber transitions.

Initially, we looked at planar fronts (using a model in one-space dimension with concentrations specified at an exposed face). Preliminary analysis indicates that, multiple fronts can propagate. That is, the phenomenon is contained in our equations with physically reasonable parameters. Understanding and modeling the physics at moving and free boundaries is still to be done. However, central problems emerging from this work are extremely difficult moving boundary problems.

Certain polymer-penetrant problems give rise to unusual nonlinear, non-Fickian diffusion alone or in combination with mechanical relaxation and/or reaction. The unusual nature of these new problems comes from the form of the conditions at fixed and moving boundaries. Preliminary results obtained by T. P. Witelski in his thesis research for D. S. Cohen indicated that evolution equations with interesting time-dependent forcing account for the propagation of sharp interfaces and the formation of shocks. This time dependence is introduced from the 
original boundary conditions even when there is no time-dependent forcing in the original equations. We have pursued this and studied the process by which the time dependence causes subtle changes in the shock formation process, including the creation of "forbidden regions" where shocks are expected from the more usual studies of reaction-diffusion equations subject to standard mathematical boundary conditions, but where they can not form in the present problems. This will have serious implications with regard to the fabrication and design of many polymeric materials.

At a fundamental level, gel systems are a unique vehicle for the study of nonlinear diffusion and phase transition dynamics. At the practical level, swelling in filled elastomers and smart and super-absorbing gels are the basis for many new emerging technologies utilizing chemical memories, chemical mechanical transducers, and novel separation schemes. D.S. Cohen and S. Xiong have formulated models for nonlinear diffusion in polymers under various combinations of twisting, compression, and stretching and are developing qualitative, quantitative, and numerical procedures for extracting theoretical and practical information from the models.

Active transport in diffusion-dominated systems also was the focus of research in this project. In subcellular biology, transport of motor molecules along polymeric pathways has recently come under experimental and theoretical scrutiny. Simultaneously, new technologies for transport in mesoscopic systems have been proposed, possibly as the basis of separation methods complementary to electrophoresis. What these topics share in common is the interplay among anisotropy, nonequilibrium forces, and strong thermal fluctuations to produce systematic motion. Our studies of mathematical and numerical methods for models of these phenomena have resulted in new insights to the application of these ideas to DNA motors and possibly to the bacteria flagella motor (both rotary in nature). A central activity associated with this project was the organization of a workshop entitled "Fluctuation Dominated Transport Processes" with the aim of bringing together the bioscientists and the condensed-matter and mathematical scientists who have been approaching these common ideas from the viewpoints of different applications.

\section{References}

1. Vieth, W.R., "Diffusion in and Through Polymers: Principles and Applications," Oxford Univ. Press, 1991.

2. Verngnaud, J. M., "Liquid Transport Processes in Polymeric Materials: Modeling and Industrial Applications," Prentice Hall, 1991.

3. Tarche, P. J., "Polymers for Controlled Drug Deliveries," CRC Press, 1991. 
4. "Abstracts of the Symposium on Sorportion and Diffusion in Glassy Polymers, Second Topical Conference on Emerging Technologies in Materials," AIChE Annual Meeting, San Francisco, 1989.

5. Cohen, D.S. and A. B. White, Jr., "Sharp Fronts due to Diffusion and Viscoelastic Relaxation in Polymers," SIAM J. Appl. Math., 51, 472-483 (1991).

6. Cohen, D.S. and A.B.White, Jr., "Sharp Fronts due to Diffusion and Stress at the Glass Transition in Polymers," J. Polymer Sci., Part B: Polymer Physics, 27, 1731-1747 (1989).

7. Knauss, W.G. and V. H. Kenner, "On the Hygrothermomechanical Characterization of Polyvinyl Acetate," J. Appl. Phys., 51, 5131-5136 (1980).

8. Losi, G. V. and W.G. Knauss, "Thermal Stress in Nonlinearily Viscoelastic Solids," J. Appl. Mech., (in press).

9. Losi, G. V. and W.G. Knauss, "Free Volume Theory and Nonlinear Thermoviscoelasticity," Polymer Sci. and Eng., (in press).

10. Martuscelli, E. and C. Marchetta, eds., "New polymeric materials: Reactive Processing and Physical Properties, Proceedings of International Seminar," June9-13, 1987, Naples, Italy. VNU Science Press, Utrecht, The Netherlands, 1987.

11. Thomas, N. and A.H. Windle, "Transport of Methanol in Poly-(Methyl Methacrylate)," Polymer, 19, 255-265 (1978).

12. Thomas, N. nd A.H. Windle, "A Theory of Case II Diffusion," Polymer, 23, 529-542 (1982).

13. Durning, C.J., "Differential Sorption in Viscoelastic-Fluids," J. Polymer Sci., Polymer Phys. Ed., 23, 1831-1855 (1985).

14. Crank, J.,"The Mathematics of Diffusion," 2nd edition, Oxford, 1976.

15. Frisch, H. L., "Sorption and Transport in Glassy Polymers - a Review," Polymer Engr. and Sci., 20, 2-13 (1980).

16. Smith, T. L., and R.E. Adam, "Effect of Tensile Deformations on Gas Transport in Glassy Polymer Films," Polymer, 22, 299-304 (1981).

17. Hayes, C.K, and D.S. Cohen, "The Evolution of Steep Fronts in Non-Fickian PolymerPenetrant Systems," J. Polymer Sci., Part-B: Polymer Physics, 30, 145-161 (1992).

18 Cox , R.W., and D.S. Cohen, A Mathematical Model for Stress-Driven Diffusion in Polymers," J. Polymer Science B: Polymer Physics, 27, 589-602 (1989).

19. Cox , R.W., "A Model for Stress-Driven Diffusion in Polymers," Ph.D. thesis, California Institute of Technology, 1988.

20. Hayes, C.K, "Diffusion and Stress-Driven Flow in Polymers," Ph.D. thesis, California Institute of Technology, 1990. 
21. Cox , R.W., "Shocks in a Model for Stress-Driven Diffusion," SIAM J. Appl. Math., 50, 1284-1299 (1990).

22. Shimabukuro, S.R., "Stress Assisted Diffusion in Polymers," Ph.D. thesis, California Institute of Technology, 1990.

23. Hayes, C.K, "A New Traveling Wave Solution of Fisher's Equation with DensityDependent Diffusivity," J. Math Bio., 29, 531-537 (1991).

24. Cohen, D.S., A.B. White, and T. P. Witelski, "Two-Dimensional Front Tracking for Diffusive Penetration of Glassy Polymers," SIAM J. Appl. Math., (in press).

25. Amann, H., "Highly Degenerate Quasilinear Parabolic Systems," Scuola Normale Superiore, 43, 135-166 (1991).

26. Amann, H., "Global Existence for a Class of Highly Degenerate Parabolic Systems," Japan J. Industrial Appl. Math., (in press).

\section{Publications}

Timothy Elston, "Fluctuation Dominated Transport," Los Alamos Report LA-UR 95-1676 (1995).

Jason Riordan, Charles Doering, and Daniel Ben-Avraham, "Fluctuations and Stability of Fisher Waves," Physical Review Letters, 75, 565 (1995).

Hans Frauenfelder, "Ligand Binding to Heme Proteins," in Proceedings of the Conference on Quantitative Biophysics at the Molecular and Mesoscopic Scale, Trieste, Italy, July 1-10, 1995, Los Alamos Report LA-UR 95-1893 (1995).

Charles Doering, Jason Riordan, and Daniel Ben-Avraham, "Fluctuations and Stability of Fisher Wavefronts," Los Alamos Report LA-UR 94-4324 (1994).

Charles Doering, Bard Ermentrout, and George Oster, "Rotary DNA Motors," Biophysical Journal (submitted), Los Alamos Report LA-UR 95-1460 (1995).

Dante Chialvo and Mark Millonas, "How Swarms Build Cognitive Maps," NATO ASI on Biology and Technology of Intelligent Autonomous Agents, Los Alamos Report LA-UR 944376 (1994). 